Arq. Bras. Med. Vet. Zootec., v.67, n.4, p.1105-1110, 2015

\title{
Eficiência produtiva ao desmame de vacas Nelore criadas no Pantanal
}

[Productive efficiency to weaning of Nellore cows raised in the Pantanal]

\author{
R.M. Silva ${ }^{1}$, J.C. Souza ${ }^{2}$, H.J. Fernandes ${ }^{3}$, U.G.P. Abreu ${ }^{4}$, P.B. Ferraz Filho ${ }^{5}$, A.N. Rosa ${ }^{6}$ \\ ${ }^{1}$ Universidade Federal de Mato Grosso do Sul - UFMS/FAMEZ - Campo Grande, MS \\ ${ }^{2}$ Universidade Federal de Mato Grosso do Sul - UFMS/CPAR - Paranaíba, MS \\ ${ }^{3}$ Universidade Estadual de Mato Grosso do Sul - UEMS/UUA - Aquidauana, MS \\ ${ }^{4}$ Embrapa Pantanal - CPAP - Corumbá, MS \\ ${ }^{5}$ Universidade Federal de Mato Grosso do Sul - UFMS/CEUL - Três Lagoas, MS \\ ${ }^{6}$ Embrapa Gado de Corte - CNPGC - Campo Grande, MS
}

\begin{abstract}
RESUMO
O trabalho objetivou avaliar a eficiência produtiva de vacas ao desmame de acordo com seu tamanho (peso) e estimar correlação fenotípica entre as características peso do bezerro ao desmame (PBD), peso da vaca ao desmame do bezerro (PVD) e relação desmame (RD = PBD/ PVD) de animais da raça Nelore criados no Pantanal de Mato Grosso do Sul. Foram utilizadas 706 observações para pares de dados de pesos de vacas e de bezerros da raça Nelore ao desmame criados em regime de pastejo. Foram formados seis grupos com base no peso de vaca ao desmame (GPVD) a partir dos desvios $(40,73 \mathrm{~kg}) \mathrm{de}$ peso em relação à média geral do rebanho $(420,56 \mathrm{~kg})$. Os grupos de vacas com menores pesos obtiveram maiores RD. As correlações fenotípicas entre PVD e PBD variaram de 0,05 a 0,26, enquanto para PVD e RD as correlações foram negativas e com variação de 0,11 a 0,25 . As correlações fenotípicas entre PBD e RD apresentaram-se positivas e de alta magnitude $(0,91$ a 0,98$)$. As maiores eficiências produtivas foram determinadas para vacas de pequeno a médio porte $(327,20$ a $438,62 \mathrm{~kg})$, além de estas serem menos exigentes quanto a nutrientes digestíveis totais (NDT). A seleção de vacas de tamanho médio (379, 83 a $461,29 \mathrm{~kg}$ ) apresenta-se mais vantajosa por gerar menor custo de mantença que as vacas maiores e produzir bezerros mais pesados que as vacas menores.
\end{abstract}

Palavras-chave: zebu, correlação fenotípica, tamanho de vaca

\begin{abstract}
The study aimed to evaluate the productive efficiency of cow at weaning according to their size (weight) and estimated phenotypic correlation between the characteristics of weaning weight of calf and cow and weaning ratio of Nellore cattle raised in the Pantanal of Mato Grosso do Sul. 706 observations were used to weight data pairs of cow and calves at weaning. Six groups were formed based on the weight of cow at weaning from deviations $(40.73 \mathrm{~kg})$ of weight in relation to the overall mean of the herd $(420.56 \mathrm{~kg})$. The groups of cows with lower weaning weight have the highest weaning ratio. Phenotypic correlations of cow weight at weaning and weaning of calf weight ranged from 0.05 to 0.26 , cow weight at weaning and weaning ratio were negative correlations ranging from -0.11 to -0.25 . Phenotypic correlations between weaning of calf and weaning ratio were positive and of high magnitude (0.91 to 0.98). The highest efficiencies were determined for cows between 327.20 and $438.62 \mathrm{~kg}$, and are less demanding on total digestible nutrients (TDN). A selection of medium sized cows $(379,83$ to $461.29 \mathrm{~kg}$ ) appears more interesting because it has lower maintenance costs than larger cows, and produces heavier calves than the smaller cows.
\end{abstract}

Keywords: zebu, phenotypic correlation, size of cow

Recebido em 3 de junho de 2013

Aceito em 12 de março de 2015

E-mail: rosana.msilva@uol.com.br 


\section{INTRODUÇÃO}

$\mathrm{Na}$ bovinocultura de corte, a eficiência do processo produtivo das matrizes reflete diretamente na base do sistema de produção. Matrizes que não parem ou que desmamam bezerros com pesos muito abaixo da média têm o mesmo custo de produção de outras que pariram e/ou que desmamaram bezerros mais pesados, não sendo, portanto, vantajosas ao sistema de produção. Nesse sentido, a razão do peso do bezerro pelo peso da vaca tem sido uma ótima ferramenta para avaliar a eficiência das fêmeas por indicar a produção e a contribuição da vaca a cada produto desmamado.

O tamanho corporal da vaca apresenta efeitos biológicos e econômicos sobre a eficiência da produção animal. Dentre os efeitos biológicos destacam-se: custo extra de energia para manutenção; associação com varias medidas de quantidade e qualidade da carne; adaptabilidade às condições de alimentação, ao clima e a outros recursos do sistema de produção (Lanna e Packer, 1997; Dias et al., 2008). Já o peso do bezerro é de grande importância no processo seletivo dos animais por representar a capacidade de crescimento do indivíduo, em conjunto com a capacidade materna para produção de leite, bem como a possibilidade de apresentar correlação fenotípica e genética positiva com pesos às idades subsequentes (Bergmann, 2003).

A seleção fenotípica para características como tamanho da vaca, peso ao desmame do bezerro e relação desmame é importante não somente pelos reflexos na sua expressão, como também na de outras que lhes são dependentes, em maior ou menor grau, podendo estes serem verificados por meio das correlações (Pereira, 2000; Dias et al., 2008).

Nesse sentido, o objetivo deste trabalho foi avaliar a eficiência produtiva de vacas ao desmame de acordo com seu tamanho (peso) e estimar a correlação fenotípica entre as características peso do bezerro ao desmame (PBD), peso da vaca ao desmame (PVD) e relação desmame (RD) de animais da raça Nelore criados no Pantanal de Mato Grosso do Sul, para diferentes grupos de peso das vacas ao desmame (GPVD).

\section{MATERIAL E MÉTODOS}

Foram utilizadas 706 observações de pares de dados para pesos de vacas e de bezerros e bezerras da raça Nelore, ao desmame, criados em regime de pasto, fornecidos por três fazendas dos municípios de Aquidauana, Miranda e Corumbá, localizados na região do Pantanal do Mato Grosso do Sul.

Os grupos de vacas foram formados com base nos desvios $(40,73 \mathrm{~kg})$ de peso em relação à média geral do rebanho $(420,56 \mathrm{~kg})$, sendo o GPVD1 o grupo de vacas com peso igual ou inferior a dois desvios abaixo da média (< $339,10 \mathrm{~kg}$ ); o GPVD2 o grupo de vacas com intervalo de peso maior que dois desvios abaixo da média e menor ou igual a um desvio abaixo da média $(339,10$ a $379,83 \mathrm{~kg})$; o GPVD3 o grupo de vacas com intervalo de peso maior que um desvio abaixo da média e menor ou igual à média (379,84 a 420,56kg); o GPVD4 o grupo de vacas com intervalo de peso maior que a média e menor ou igual a um desvio acima da média (420,57 a 461,29kg); o GPVD5 o grupo de vacas com intervalo de peso maior que um desvio acima da média e menor ou igual a dois desvios acima da média (461,30 a 502,02kg); e o GPVD6 o grupo de vacas com peso maior que dois desvios acima da média (> 502,02).

A relação peso do bezerro e peso da vaca foi estimada por meio da expressão:

$$
\mathrm{RD}=\mathrm{PBD} / \mathrm{PVD} \text {, }
$$

em que $\mathrm{RD}=$ relação desmame PBD = peso do bezerro ao desmame e PVD = peso da vaca ao desmame.

Com base no NRC (2000), estimaram-se as médias de nutrientes digestíveis totais (NDTm) necessários à mantença de animais da raça Nelore de acordo com seu peso vivo, por meio da equação:

$\mathrm{NDTm}=(\mathrm{EMm} / 0,82) / 4,409$,

sendo EMm a energia média metabolizável, dada pela seguinte expressão: $\mathrm{EMm}=([$ peso médio do animal] $\left.{ }^{0,75} * 0,07\right) / 0,64$.

As análises descritivas foram realizadas utilizando-se o pacote estatístico SAS (2011), e as análises de correlação fenotípica foram feitas por meio do procedimento CORR. 


\section{RESULTADOS E DISCUSSÃO}

Os diferentes grupos de peso de vaca apresentam uma proporção, em relação à média geral do rebanho $(420,56 \mathrm{~kg})$, de $78 \%, 87 \%, 95 \%, 104 \%$,
$113,42 \%$ e $123,81 \%$ para GPVD1, GPVD2, GPVD3, GPVD4, GPVD5 e GPVD6, respectivamente, conforme apresentado na Tab. 1.

Tabela 1. Médias e desvio-padrão (DP) de peso da vaca ao desmame (PVD), peso do bezerro ao desmame (PBD) e relação desmame (RD)

\begin{tabular}{clll}
\hline \multirow{2}{*}{ GPVD } & PVD & PBD & RD \\
\cline { 2 - 4 } & Média \pm DP & & \\
\hline 1 & $327,20 \pm 11,01$ & $185,60 \pm 22,73$ & $0,57 \pm 0,07$ \\
2 & $364,81 \pm 11,17$ & $188,73 \pm 33,52$ & $0,52 \pm 0,09$ \\
3 & $400,04 \pm 11,34$ & $192,27 \pm 29,73$ & $0,48 \pm 0,08$ \\
4 & $438,62 \pm 11,94$ & $198,05 \pm 25,89$ & $0,45 \pm 0,06$ \\
5 & $477,01 \pm 11,72$ & $201,89 \pm 24,71$ & $0,42 \pm 0,05$ \\
6 & $520,00 \pm 18,24$ & $206,24 \pm 19,26$ & $0,40 \pm 0,04$ \\
\hline
\end{tabular}

Total 420,56 $440,73 \quad 195,27 \pm 28,41 \quad 0,47 \pm 0,08$

GPVD: grupo de peso de vaca ao desmame. 1: vacas com 339,10kg ou menos; 2: vacas entre 339, 10 e $379,83 \mathrm{~kg}$; 3 : vacas entre 379,84 e 420,56kg; 4: vacas entre 420,57 e 461,29kg; 5 : vacas entre 461,30 e 502,02kg; e 6: vacas acima de $502,02 \mathrm{~kg}$.

Com base, principalmente, no reflexo financeiro dentro do sistema de produção, ressalta-se o questionamento sobre o tamanho de vaca ideal. Nesse sentido, é oportuno avaliar algumas considerações quanto à receita oriunda da venda de bezerros, em que diferenças no tamanho de vaca dentro do rebanho podem gerar aumento ou redução da produtividade por área, além de que o comércio de bezerros no Pantanal baseia-se na unidade de bezerro desmamado, com certa variação do preço de acordo com o desempenho desses animais.

Considerando-se índices zootécnicos como taxa de desmame de $59 \%$ e taxa de lotação em 0,4 unidade animal (UA) por hectare (ha), determinados por Carvalho et al. (2009) para rebanhos Nelore na região de Aquidauana, estado de Mato Grosso do Sul, uma das regiões com rebanhos avaliados neste estudo, é possível observar a influência do tamanho da vaca sobre o retorno econômico de um sistema de cria, ao simular um rebanho com 100 vacas com média de peso de 520kg (GPVD6), em uma área de 287,5ha, com produção de 59 bezerros desmamados com 206,24kg, o que gerou um total $(206,24 \mathrm{~kg} * 59$ bezerros) de $12.168,16 \mathrm{~kg}$ de bezerro desmamado ao ano.

Já com um rebanho de vacas com peso médio de 400,04kg (GPVD3) nessa mesma área (287,5 ha) e nas mesmas condições (0,4 UA/ha), podem-se produzir 129 vacas e, por meio desta, obter 76 bezerros desmamados com $192,27 \mathrm{~kg}$, considerando a mesma taxa de desmame $(59 \%)$. O resultado $(192,27 \mathrm{~kg} * 76)$ é um total de $14.612,52 \mathrm{~kg}$ de bezerro desmamado ao ano. De acordo com esse exemplo, pode-se observar uma diferença de 17 bezerros produzidos e $2.444,05 \mathrm{~kg}$ de bezerro desmamado, devido ao tamanho da vaca a ser utilizada no rebanho.

Outro ponto a ser observado é quanto à equivalência na renda originária das vacas no momento do descarte. No caso de vacas mais pesadas (GPVD6), considerando uma taxa de $20 \%$ de descarte do total de 100 vacas, serão descartados $10.400 \mathrm{~kg}$ de vaca ao ano $(20 * 520 \mathrm{~kg})$, enquanto para vacas médias (GPVD3) esse descarte passa a ser de $(26 * 400,04 \mathrm{~kg}) 10.401,04 \mathrm{~kg}$ de vaca ao ano, considerando a mesma taxa de $20 \%$ de descarte do rebanho de 129 vacas.

Stewart e Martin (1983) já haviam constatado que o aumento no peso adulto das vacas aumenta o peso médio de desmame dos bezerros, porém verificaram uma redução no número de bezerros por quilograma de vaca, no total de bezerro desmamado e no tempo de permanência da vaca no rebanho. 
Como o custo de produção de uma vaca está relacionado diretamente ao seu tamanho (peso), é vantajoso que esta vaca desmame bezerros com uma boa proporção de seu peso, já que a renda do sistema de cria é atribuída, principalmente, à produção de bezerros, além do descarte de vacas (Brinks et al., 1962; Barbosa et al., 2010). Kress et al. (2001) afirmam que essa relação é um indicador de eficiência dos desempenhos produtivo e econômico do rebanho, já que essa característica pode gerar uma elevação do custo de produção por área.

Dinkel e Brown (1978), ao trabalharem com animais da raça Angus, cujas vacas e cujos bezerros pesaram, em média, $463,1 \pm 37,45 \mathrm{~kg}$ e $217,70 \pm 27,67 \mathrm{~kg}$, respectivamente, determinaram uma relação desmame de 0,47 . Considerando o intervalo de peso correspondente a essa média, a relação desmame apresenta-se superior ao determinado neste estudo. Deve-se considerar que as diferenças encontradas na literatura podem estar muito relacionadas a questões de ambiente e também genéticas.

Os dados revelam que, a partir do GPVD3, houve um aumento do peso do bezerro desmamado, porém uma diminuição na relação desmame. Johnson et al. (2007) relataram que vacas com relação desmame acima de 0,50 são mais eficientes produtiva e economicamente que vacas com relação desmame abaixo de 0,50.

Silva et al. (2000), em estudos com animais Nelore, determinaram médias de peso e erropadrão para vacas e bezerros ao desmame, respectivamente, de $479,20 \pm 1,60 \mathrm{~kg}$ e $179,00 \pm 0,90 \mathrm{~kg}$ para rebanho sem seleção, de $484,50 \pm 1,20 \mathrm{~kg}$ para vaca, e de $180,30 \pm 0,70 \mathrm{~kg}$ para rebanho selecionado para peso pósdesmame. Ambos os resultados apresentam uma relação desmame de 0,37 , que, em comparação com a relação encontrada para o GPVD5, na qual se enquadra o peso das matrizes analisadas, apresenta-se $12 \%$ inferior aos resultados estimados neste estudo.

Vacas da raça Aberdeen Angus e 1/2 Angus x 1/2 Nelore, com médias de peso ao desmame de
$309,4 \pm 13,0 \mathrm{~kg}$ e de $313,5 \pm 9,2 \mathrm{~kg}$, desmamaram, segundo Ribeiro et al. (2001), bezerros com médias de peso iguais a $123,7 \pm 5,6 \mathrm{~kg}$ e $131,8 \pm 4,0 \mathrm{~kg}$, respectivamente, o que representa uma relação desmame de 0,40 e 0,42, abaixo da citada neste trabalho, para GPVD1, correspondente a esse intervalo de peso. A relação desmame para todas as classes estudadas apresenta-se superior às determinadas por Sala $e t$ al. (2009) para animais também da raça Nelore, cujas vacas, com média de peso de $508,91 \pm 1,04 \mathrm{~kg}$ e $445,42 \pm 1,69 \mathrm{~kg}$, apresentaram relação desmame de aproximadamente 0,38.

Perotto (1999) relatou que o maior tamanho (peso) da vaca em ambientes em que a disponibilidade de alimento não supre as necessidades dos animais pode resultar em baixa eficiência produtiva, refletindo na produção de bezerros mais leves ao desmame. Para Barbosa (2006), vacas de tamanhos médios e grandes são mais eficientes do que as vacas de tamanho pequeno, quando as condições de alimentação e manejo não são limitantes, ou seja, cada região influenciará em diferentes resultados.

Tendências semelhantes foram também verificadas por Ribeiro et al. (2001), os quais verificaram exigências de nutrientes digestíveis totais (NDT) $11,4 \%(5,85$ vs. 6,60) maiores para as vacas da raça Charolesa em comparação às fêmeas da raça de menor porte (Aberdeen Angus). Por outro lado, os autores verificaram que, apesar do menor porte, as vacas da raça Angus desmamaram, em média, 21,5\% mais quilos de bezerro para cada $100 \mathrm{~kg}$ de vaca ao parto $(42,0$ vs. $34,6 \mathrm{~kg})$ e $15,7 \%$ mais quilos de bezerro para cada $100 \mathrm{~kg}$ de vacas ao desmame $(41,2$ vs. $35,6 \mathrm{~kg})$.

Ao se utilizar a média de peso de cada grupo de vaca e bezerro, pode-se verificar a quantidade média de energia metabolizável (EMm) e de nutrientes digestíveis totais (NDTm) necessária diariamente para cada grupo de vaca (Tab. 2). Por meio desses, verifica-se, por exemplo, que a diferença de exigências de NDTm entre o GPVD1 e o GPVD2 corresponde a $28 \%$. 
Eficiência produtiva...

Tabela 2. Energia necessária para mantença das vacas da raça Nelore de acordo com o peso médio ao desmame

\begin{tabular}{cccc}
\hline GPVD & Peso $(\mathrm{kg})$ & EMm $($ Mcal/dia $)$ & NDTm $(\mathrm{kg} / \mathrm{dia})$ \\
\hline 1 & 327,2 & 8,4 & 2,3 \\
2 & 364,8 & 9,1 & 2,5 \\
3 & 400,0 & 9,7 & 2,7 \\
4 & 438,6 & 10,4 & 2,9 \\
5 & 477,0 & 11,1 & 3,0 \\
6 & 520,0 & 11,9 & 3,2 \\
\hline Média & 420,5 & 10,1 & 2,8
\end{tabular}

GPVD: grupo de peso de vaca ao desmame. 1: vacas com 339,10kg ou menos; 2: vacas entre 339,10 e 379,83kg; 3 : vacas entre 379,84 e $420,56 \mathrm{~kg}$; 4 : vacas entre 420,57 e $461,29 \mathrm{~kg}$; 5 : vacas entre 461,30 e 502,02kg e 6: vacas acima de $502,02 \mathrm{~kg}$.

Considerando animais criados na planície pantaneira, onde a oferta de pastagem não é tão grande quanto no planalto, em áreas formadas, essa informação torna-se relevante, a ponto de demonstrar que vacas mais pesadas não são eficientes por unidade de área, tendo em vista o sistema de cria.

Ao se analisarem os coeficientes de correlação fenotípica, nota-se que, entre o peso da vaca ao desmame e o peso do bezerro desmamado, as correlações foram, em sua maioria, de baixa magnitude, indicando uma possível ausência de associação entre essas características.

Correlações positivas e maiores que as demonstradas neste estudo (Tab. 3), entre PDB e $\mathrm{RD}$, foram apresentadas por Dinkel e Brown (1978) com magnitudes de 0,55. Já para a correlação entre PVD e PBD $(0,06)$, os valores estão em consonância com os determinados para PVD, além de correlações negativas entre PVD e RD de $-0,49$, em se tratando, segundo os autores, de um rebanho de animais Angus e Charolês criados em confinamento nos Estados Unidos. Já Restle et al. (2005), ao avaliarem animais da raça Nelore, estimaram correlações fenotípicas entre PVD e PBD de 0,75 .

O PVD não está correlacionado positivamente à $\mathrm{RD}$, sugerindo que, apesar de vacas maiores produzirem bezerros grandes, não há como afirmar que esse apresente uma alta relação de quilo de bezerro desmamado. Correlações fenotípicas de alta magnitude e positivas foram encontradas somente para as características de $\mathrm{PBD}$ em relação à $\mathrm{RD}$, visto que quanto maior o numerador, sendo este o peso do bezerro desmamado, maior a relação desmame.
Tabela 3. Correlações fenotípicas entre as características de peso da vaca ao desmame (PVD), peso de bezerro ao desmame (PBD) e relação desmame (RD) para diferentes classes de peso de vacas

\begin{tabular}{ccc}
\hline PVD & PBD & RD \\
\hline PVD $^{1}$ & 0,07 & $-0,21$ \\
PVD $^{2}$ & 0,06 & $-0,11$ \\
PVD $^{3}$ & 0,05 & $-0,22$ \\
PVD $^{4}$ & 0,05 & $-0,25$ \\
PVD $^{5}$ & 0,07 & $-0,13$ \\
PVD $^{6}$ & 0,29 & $-0,12$ \\
\hline PBD $^{1}$ & & 0,95 \\
PBD $^{2}$ & & 0,98 \\
PBD $^{3}$ & & 0,98 \\
PBD $^{4}$ & & 0,98 \\
PBD $^{5}$ & & 0,98 \\
PBD $^{6}$ & & 0,91 \\
\hline
\end{tabular}

1: vacas com $339,10 \mathrm{~kg}$ ou menos; 2: vacas entre 339,10 e $379,83 \mathrm{~kg}$; 3 : vacas entre 379,84 e $420,56 \mathrm{~kg}$; 4: vacas entre 420,57 e $461,29 \mathrm{~kg}$; 5: vacas entre 461,30 e $502,02 \mathrm{~kg}$ e 6 : vacas acima de $502,02 \mathrm{~kg}$.

\section{CONCLUSÕES}

Apesar de as vacas de menor porte demonstrarem maior eficiência quanto à relação desmame, a seleção de vacas de tamanho médio $(379,83$ a $461,29 \mathrm{~kg})$ apresenta-se como mais vantajosa por gerar menor custo de mantença que as vacas maiores e produzir bezerros mais pesados que as vacas menores (em média, 192 a $198 \mathrm{~kg}$ ). 


\section{AGRADECIMENTOS}

Ao M.e Eduardo S. Corrêa, à fazenda Boa Sorte (Miranda-MS) e às instituições UEMS, Embrapa Pantanal, Capes e FUNDECT, pelo apoio ao desenvolvimento deste estudo.

\section{REFERÊNCIAS}

BARBOSA, P.F. Tamanho estrutural corporal e desempenho produtivo de bovinos de corte. In: REUNIÃO ANUAL DA SOCIEDADE BRASILEIRA DE ZOOTECNIA, 43., 2006, João Pessoa. Anais... Paraíba: [SBZ] 2006. (Resumo).

BARBOSA, F.A.; GRAÇA, D.A.; ANDRADE, V.J. et al. Produtividade e eficiência econômica de sistemas de produção de cria, recria e engorda de bovinos de corte na região sul do estado da Bahia. Arq. Bras. Med. Vet. Zoot., v.62, p.677685,2010

BERGMANN, J.A.G. Objetivos e critérios de seleção. In: WORKSHOP DE SELEÇÃO EM BOVINOS DE CORTE, 4., 2003, Ribeirão Preto. Anais... São Paulo: [s.n.], 2003. p.8. (Resumo).

BRINKS, J.S.; CLARK, R.T.; KIEFFER, N.M.; QUESENBERRY, J.R. Mature weight in Hereford range cows - heritability, repeatability and relationship to calf performance. J. Anim. Sci., v.21, p.501, 1962.

CARVALHO, T.B.; ABREU, U.G.P.; ALMEIDA, B.S. et al. Custo de produção em pecuária de corte em 2009, na região do Pantanal de Aquidauana (MS). Corumbá: Embrapa Pantanal, 2009. Disponível em: <http://www.cpap.embrapa.br/publicacoes/ download.php?arq_pdf $=$ COT77 $>$. Acessado em: 3 mar. 2013.

CHANGES and enhancement through: release 8.2. Cary, N.C: SAS Institute, 2011.

DIAS, J.C.; ANDRADE, V.J.; MARTINS, J.A.M. et al. Correlações genéticas e fenotípicas entre características reprodutivas e produtivas de touros da raça Nelore. Pesqui. Agropecu. Bras., v.43, p.53-59, 2008.

DINKEL, C.A.; BROWN, M.A. An evaluation of the ratio of calf weight to cow weight as an indicator of cow efficiency. J. Anim. Sci., v.46, p.614-617, 1978.
JOHNSON, Z.B.; BROWN JR., A.H.; DEWEY S.T. A Comparison of weaning ratio in five breeds of cattle. Ark. Agric. Exp. Stn. Res.. v.553, p.10-12, 2007.

KRESS, D.D.; ANDERSON, D.C.; STEVENS, J.D. et al. Calf weight/cow weight ratio of weaning as a predictor of beef cow efficiency. In: AMERICAN SOCIETY OF ANIMAL SCIENCE, 52., 2001, Western Section. Proceedings... Westem Section:. ASAS: 2001. (Resumo). p.130.

LANNA, D.P.; PACKER, I.U. A produtividade da vaca nelore. In: SIMPÓSIO: O NELORE DO SÉCULO XXI, 4., 1997, Uberaba. Anais... Uberaba: [s.n.] 1997. p.73. (Resumo).

NUTRIENTS requeriments of beef cattle. 7.ed. Washington, D.C.: NRC, 2000. 244p.

PEREIRA, J.C.C. Contribuição genética do zebu na pecuária bovina do Brasil. Inf. Agropecu., v.21, p.30-38, 2000.

PEROTTO, D. Uso de matrizes selecionadas e habilidade materna. In: SIMPÓSIO DE GENÉTICA E MELHORAMENTO ANIMAL, 1., 1999, Palotina. Anais... Palotina: [s.n.] 1999. p.98.

RESTLE, J.; PACHECO, P.S.; PADUA, J.T. et al. Efeitos d taxa de ganho de peso pré-desmama de bezerras de corte e do nível nutricional pósparto, quando vacas, sobre a produção e composição do leite e o desempenho de bezerros. Rev. Bras. Zootec., v.34, p.197-208, 2005.

RIBEIRO, E.L.A.; RESTLE, J.; ROCHA, M.A. et al. Eficiência produtiva em vacas primiparas das raças Aberdeen Angus e Charolês. Rev. Bras. Zootec., v.30, p.125-132, 2001.

SALA, V.E.; ALBUQUERQUE, L.G.; MERCADANTE, M.E.Z. et al. Eficiência produtiva em vacas da raça Nelore. Bol. Ind. Anim., v.66, p.107-113, 2009.

SILVA, J.A.II.V.; RAZOOK, A.G.; TONHATL, H.; FIGUEIREDO, L.A. Efeito da seleção para peso pós-desmama sobre indicadores da eficiência produtiva de vacas da raça Nelore. Rev. Bras. Zootec., v.29, p.1020-1027, 2000.

STEWART, T.S.; MARTIN, T.G. Optimal mature size of Angus cows for maximum cow productivity. Anim. Prod., v.37, p.179-182, 1983. 